\title{
Evaluación de la Atención Integral al Pos-aborto en 13 Hospitales de Colombia
}

\section{Evaluating post-abortion care in 13 Colombian hospitals}

Pío I. Gómez-Sánchez ${ }^{1}$, Inés Escandón ${ }^{2}$ y Hernando Gaitán-Duarte ${ }^{1}$ 1. Departamento de Obstetricia y Ginecología, Facultad de Medicina, Universidad Nacional de
Colombia pigomezs@unal.edu.co, hggaitand@cable.net.co

2. EngenderHealth, New York, NY. iescandon@engenderhealth.org

Recibido 18 Septiembre 2006/ Enviado para Modificación 16 Marzo 2007/Aceptado 25 Abril 2007

\section{RESUMEN}

Objetivos El propósito de este trabajo es describir la experiencia con la estrategia de Atención Integral al Posaborto (APA) en 13 hospitales Colombianos en lo referente a tres componentes: Consejería, tratamiento y planificación familiar.

Métodos Estudio mixto con un componente cuantitativo (corte transversal) y otro cualitativo. Población: Hospitales en Colombia que recibieron capacitación en APA entre 1999 y 2002 y que cumplieron con criterios de inclusión y exclusión. Se hizo un muestreo secuencial por conveniencia, entrevistando 127 pacientes, 13 gerentes, y 39 proveedores.

Resultados Entre 1999 y 2002 se atendieron 26199 mujeres con aborto incompleto. Se trataron con legrado uterino por aspiración manual (LUA) el $40 \%$ y con legrado uterino con cureta (LUC) el $60 \%$. En los casos de LUA se brindó consejería en el $99,8 \%$ y en los de LUC en $57,3 \%$. Utilizando LUA no hubo perforaciones uterinas aunque hubo 2 reacciones vagales mientras con LUC hubo 9 perforaciones uterinas (Chi2: 2,16; $p=0,14)$. Con LUC hubo necesidad de reaspiración por evacuación incompleta en 13 casos mientras que en LUA en un caso (Chi2: 6,25 p=0,01). Las pacientes sometidas a LUA refirieron haber estado muy satisfechas y satisfechas en más del $90 \%$ mientras que para LUC el grado de satisfacción fue del $60 \%$. Manifestaron satisfacción con la estrategia APA todos los proveedores y 12 de 13 gerentes.

Conclusiones La técnica LUA presentó menor morbilidad y mayor satisfacción en las usuarias. La mayoría de los gerentes y la totalidad de los proveedores manifestaron satisfacción con la estrategia APA.

Palabras Clave: Atención, aborto, anticoncepción, evaluación, Colombia(fuente: DeCS, BIREME).

\section{ABSTRACT}

Objectives This work describes Post-abortion Care strategy (PAC) experience in 13 Colombian hospitals regarding three components (counselling, suitable treatment and family planning). 
Methods The study had quantitative (cross-sectional study) and qualitative components. Population: Colombian hospitals receiving PAC training between 1999 and 2002 and fulfilling inclusion and exclusion criteria. Sequential convenience sampling was used; 127 patients, 13 managers and 39 health-care providers were interviewed.

Results 26,199 women were treated for incomplete abortion during the study period. $40 \%$ were treated using Manual Vacuum Aspiration (MVA) and $60 \%$ using sharp curettage (SC). Family planning counselling was provided for $99,8 \%$ of MVA cases; however, only $57,3 \%$ of SC cases received it. There were 9 uterine perforations using SC and none in MVA cases (Chi square: 2.16; $p=0.14$ ). There were 2 vagal reactions using MVA. Uterine evacuation was needed in 13 SC cases but in only one MVA case (Chi square: 6,25 $p=0.01$ ). Patient satisfaction levels with hospital treatment were evaluated: $90 \%$ of those receiving MVA treatment reported being satisfied or very satisfied, compared to just $60 \%$ of those treated with SC. All health-care providers and 12 out of 13 managers reported satisfaction with the PAC strategy and with 12 out of 13 managers as well.

Conclusions MVA treatment was associated with less treatment-related morbidity and higher levels of satisfaction amongst patients. Most managers and all healthcare providers stated their satisfaction with the PAC strategy.

Key Words: Attention, abortion, family planning, evaluation, Colombia (source; $\mathrm{MeSH}$, $N L M)$.

$\mathrm{E}$ l personal de los servicios ginecobstétricos de emergencia frecuentemente es testigo de uno de los principales problemas de salud de la mujer: el aborto y sus complicaciones. En muchos hospitales, el tratamiento de las complicaciones tales como aborto incompleto, hemorragia e infección, constituyen una gran proporción de las pacientes atendidas en los servicios de urgencias $(1,2)$.

En el mundo, 46 millones de mujeres presentan abortos cada año (3). De estas mujeres, 78 \% viven en países emergentes. La Organización Mundial de la Salud y otras instituciones estiman que en América Latina 40 de cada mil mujeres en edad reproductiva, tienen abortos inseguros (4). Éste causa seis mil muertes cada año (25\% de las muertes maternas en la región) (5). En Colombia el $24 \%$ de los embarazos termina en aborto inducido, $26 \%$ en nacimientos no deseados y un $29 \%$ de las mujeres entre 15 a 55 años, declara haber tenido aborto inducido (6).

En la actualidad el manejo del aborto contempla no solo los aspectos médicos sino también los determinantes bio-psico-sociales que conllevan a un embarazo no siempre planeado, y un mal desenlace materno o fetal. De este modo los 
factores sociales y culturales podrían tener un muy importante rol como factores asociados al aborto y sus complicaciones.(7)

El consorcio de Atención Integral al posaborto integrado por varias agencias e instituciones que trabajan en el campo de salud sexual y reproductiva ha venido trabajando en la estrategia mundial de atención integral al posaborto (APA) con cinco elementos básicos a saber:

1. Alianzas entre comunidades y proveedores de servicios: para prevenir embarazos no deseados y abortos de alto riesgo, para movilizar recursos tendientes a ayudar a que las mujeres reciban atención apropiada y tratamiento oportuno del aborto y sus complicaciones y para asegurar que los servicios de salud reflejen y satisfagan las expectativas de la comunidad y sus necesidades

2. Orientación /Consejería: para identificar y responder a las necesidades de salud emocionales y físicas así como otras necesidades y preocupaciones de las mujeres

3. Tratamiento del aborto incompleto y las complicaciones: Esto incluye técnicas de evacuación uterina sencillas, eficaces, seguras y de bajo costo. Es el caso del legrado uterino por aspiración que además de poderse realizar con anestesia local y a bajo costo, permite ser realizado en cualquier institución por no requerir instrumental sofisticado ni electricidad $(8,9)$.

4. Servicios de anticoncepción y de planificación familiar: para ayudar a las mujeres a prevenir gestaciones no deseadas y/o espaciar sus embarazos.

5. Servicios de salud sexual y reproductiva y otros servicios de salud: que preferiblemente se proporcionan en el mismo lugar o remitir a otros servicios accesibles dentro de la red de servicios.

Con el apoyo de EngenderHealth ${ }^{1}$ se brindó capacitación en Salud sexual y reproductiva (SSR) a 26 instituciones hospitalarias Colombianas con énfasis en consejería, prevención de infecciones, gerencia en SSR, anticoncepción, herramientas de calidad, atención integral al posaborto (APA), minilaparotomía para ligadura tubaria, Dispositivo intrauterino (DIU) posparto y posaborto, así como en legrado uterino por aspiración manual (LUA) y en algunos hospitales vasectomía sin bisturí.

${ }^{1}$ Nota del Editor: EngenderHealth es una ONG sin ánimo de lucro con sede en Nueva York que trabaja en varios países de África, Asia, Latinoamérica y El Caribe 
El propósito de este trabajo es evaluar la estrategia APA en los hospitales Colombianos que se capacitaron en lo referente a los componentes de consejería, tratamiento del aborto incompleto y complicaciones así como planificación familiar.

\section{MÉTODOS}

Diseño: Estudio mixto evaluativo con un componente cuantitativo (corte transversal) y otro cualitativo.

Población: Hospitales en Colombia que recibieron capacitación en Atención Integral al posaborto (APA) entre 1999 y 2002. Estos fueron: La maternidad Rafael Calvo de Cartagena, el Hospital Universitario Evaristo García de la ciudad de Cali, en Antioquia los Hospitales San Rafael de Itagüi y Marco Fidel Suárez de Bello, en Bogotá los Hospitales de Meissen, San Pablo de Fontibón, Vistahermosa, Tunal y Bosa, en Boyacá el Hospital Fructuoso Reyes de Santa Rosa de Viterbo y Hospital Regional de Duitama, en Cundinamarca los Hospitales San Rafael de Fusagasugá, San Francisco de Gachetá y el San Juan de Dios de Zipaquirá. Estas instituciones son de nivel medio y alta complejidad, atienden población de régimen subsidiado, población pobre y vulnerable y en menor proporción población de régimen contributivo.

Criterios de inclusión de las Instituciones:

Implementación del programa de APA (corroborado por la revisión de los informes mensuales de las instituciones y reporte de las visitas de seguimiento realizadas)

Criterios de Exclusión:

Cambio de gerentes y/o de personal capacitado

Reestructuración de la institución

Ausencia de informes mensuales de actividades

Muestra: Se entrevistaron mujeres que habían sido usuarias de APA (mínimo de 3 mujeres y un máximo de 10 por cada institución). En total se entrevistaron 127 mujeres. Se entrevistaron los 13 gerentes, y tres proveedores por institución (39 en total). Se realizó un muestreo secuencial por conveniencia.

Fuentes y recolección de información: Se revisaron los informes de los servicios y programáticos, informes de capacitación e informes de visitas médicas trimestrales, de los años 1999 a 2002. Cada institución enviaba un reporte cuantitativo de autodiligenciamiento en formato preestablecido que contenía 
variables a estudiar. El formato ha sido validado y utilizado en 4 países Latinoamericanos. Cada institución tenía un coordinador del programa de APA quien había sido capacitado en el diligenciamiento electrónico del reporte mensual. La visita médica fue realizada por un mismo observador (Ginecólogo), utilizando un formato precodificado que contenía variables cuantitativas y cualitativas en la observación de los servicios, así como entrevistas semiestructuradas a personal de la institución (Gerente, personal médico, de enfermería, personal auxiliar y usuarias de los servicios). El formato de visita médica también fue validado y utilizado con algunas modificaciones en varios países donde EngenderHealth apoya programas. Para la recolección de los datos cualitativos se utilizaron las técnicas de observación y entrevista semiestructurada que contenía los temas a explorar. Se hizo una prueba de campo con 10 entrevistados previa a su implementación en el estudio.

Variables consideradas:

- Número de usuarias atendidas con legrado uterino por aspiración manual (LUA). En algunos sitios se conoce como Aspiración Manual Endouterina (AMEU).

- Número de usuarias atendidas con legrado uterino con cureta cortante (LUC). - Tipo de anestesia utilizada en cada caso: técnica utilizada par abolir el dolor. Anestesia general: utilización de medicamentos intravenosos o inhalados con pérdida de la conciencia. Anestesia local: aplicación de medicamentos en el sitio operatorio con la paciente conciente.

- Número de consejerías brindadas: pacientes que recibieron asesoría sobre métodos de planificación familiar incluyendo ventajas, desventajas, costos, riesgos y efectos esperados de cada uno de ellos.

- Complicaciones (tipo y número): pacientes que presentaron perforación uterina, reacción vagal asociada a la manipulación cervical.

- Evacuación incompleta: presencia de restos ovulares después de la limpieza endouterina que se manifiesta por sangrado abundante y dolor, y es indicación de nueva evacuación.

- Percepción de satisfacción de las usuarias con la atención recibida, con las siguientes opciones: no satisfecha, poco satisfecha, satisfecha, muy satisfecha. - Percepción de satisfacción de los proveedores con el programa APA, con las siguientes opciones: no satisfechos, poco satisfechos, satisfechos, muy satisfechos.

- Usuaria: mujer que solicitó servicios de la institución por aborto incompleto y/ o sus complicaciones.

- Gerente: profesional que tiene a su cargo la dirección de la institución proveedora o profesional de la salud que participa en los servicios de atención 
posaborto y que puede ser: enfermero(a) profesional, especialista en obstetricia y ginecología, y médico(a) general.

Análisis: Se realizó análisis univariado calculando media y desviación estándar (DE) para variables cuantitativas y proporciones para las categóricas. La asociación estadística de dos o más proporciones se estimó con la prueba de Chi cuadrado. La base de datos fue creada en Microsoft Excel 2000 y fue analizada en Stata 8.0.

El análisis de la información cualitativa se realizó simultáneamente con la recolección de la información, y posteriormente teniendo en cuenta la trascripción de la entrevista y las anotaciones del entrevistador. Posteriormente codificación, clasificación de códigos y conformación de categorías. Finalmente análisis de categorías, interrelación entre ellas y triangulación entre los investigadores.

\section{RESULTADOS}

De los 26 hospitales se incluyeron 13 instituciones ya que las restantes sufrieron importantes cambios de personal y reestructuraciones administrativas que limitaron el desarrollo del programa deAPA. Entre 1999 y 2002, los 13 hospitales que cumplieron con criterios de inclusión-exclusión atendieron 26199 mujeres con aborto incompleto (Tabla 1). Se realizó legrado uterino por (LUA) en el 40 $\%$ de los casos y (LUC) en el $60 \%$.

Observamos que las mujeres a quienes se les realizó LUA tuvieron consejería en el 99,8 \% y las de LUC en 57,3\%. LUA se realizó principalmente bajo anestesia local (algunas veces con sedación leve), mientras que LUC siempre se realizó con anestesia general.

De las entrevistas a personal de las instituciones se pudo deducir que la decisión de usar una u otra técnica dependió de la preferencia del proveedor(a) en la mayoría de los casos; la excepción fue la Maternidad Calvo de Cartagena donde las mujeres reciben toda la información necesaria sobre ventajas y desventajas de las dos técnicas y realizan una verdadera elección informada. En los casos de LUA hubo 2 casos de reacción vagal, no hubo perforaciones uterinas mientras que en LUC hubo 9 (Chi2: 2,16; $\mathrm{p}=0,14$ ).

En los casos tratados con LUC se hizo reaspiración por evacuación incompleta en 13 casos mientras que en los tratados con LUA en un solo caso 
(Chi' ${ }^{2}$ 6,25 p=0,01). En los casos con LUC que se reevacuaron, hubo 3 casos de EPI y ninguno en los de LUA.

Características de las pacientes entrevistadas

Las 127 mujeres entrevistadas tuvieron un rango de edad comprendido entre los 19 y los 43 años (mediana: 24); la mitad de ellas tenían menos de 30 años. El 80 \% mantuvieron con su pareja una relación consensual o de casada en convivencia, bajo un mismo techo. El 10 \% declaró que su relación no era estable, siendo en algunos casos casual. Alrededor de cuatro de cada diez mujeres había abandonado los estudios por diversas causas, entre las que se destacan el matrimonio y/o embarazo. La mayoría tenía primaria incompleta, en menor proporción bachillerato incompleto y sólo $5 \%$ habían finalizado bachillerato. Cerca del $37 \%$ estaban insertadas al mercado laboral formal e informal, el resto estaban desempleadas y dependían económicamente del compañero.

Características de los proveedores: Los gerentes fueron 12 médicos y una enfermera. Llevaban en su cargo entre 1 a 14 años (mediana 4). De los 39 proveedores, 14 eran especialistas en Obstetricia y Ginecología, 16 eran médicos generales, 8 enfermeras profesionales y un profesional de psicología.

Análisis de las entrevistas:

Usuarias. En general, la percepción de las mujeres atendidas por aborto incompleto en todas las instituciones fue de buena calidad en la atención. Las pacientes sometidas a LUA refirieron haber estado muy satisfechas y satisfechas en más del $90 \%$ mientras que para LUC fue del $60 \%$. De cada 10 de las entrevistadas, 7 manifestaron haber usado medicamentos para inducirse el aborto, y habían obtenido el conocimiento del medicamento de amigas y la mayoría lo habían conseguido directamente en farmacias. Sólo la mitad de ellas había recibido en la consejería información sobre doble protección.

Proveedores. En la entrevista a los gerentes 12 manifestaron satisfacción con el programa APA por: haber facilitado el acceso de los servicios a la comunidad y menor tiempo de recuperación de las mujeres. Uno manifestó no estar muy satisfecho pues algunas Empresas Promotoras de Salud (EPS), habían glosado cuentas por que el bloqueo paracervical anestésico no estaba en el Manual de Procedimientos y Actividades del plan obligatorio de salud (Mapipos). 


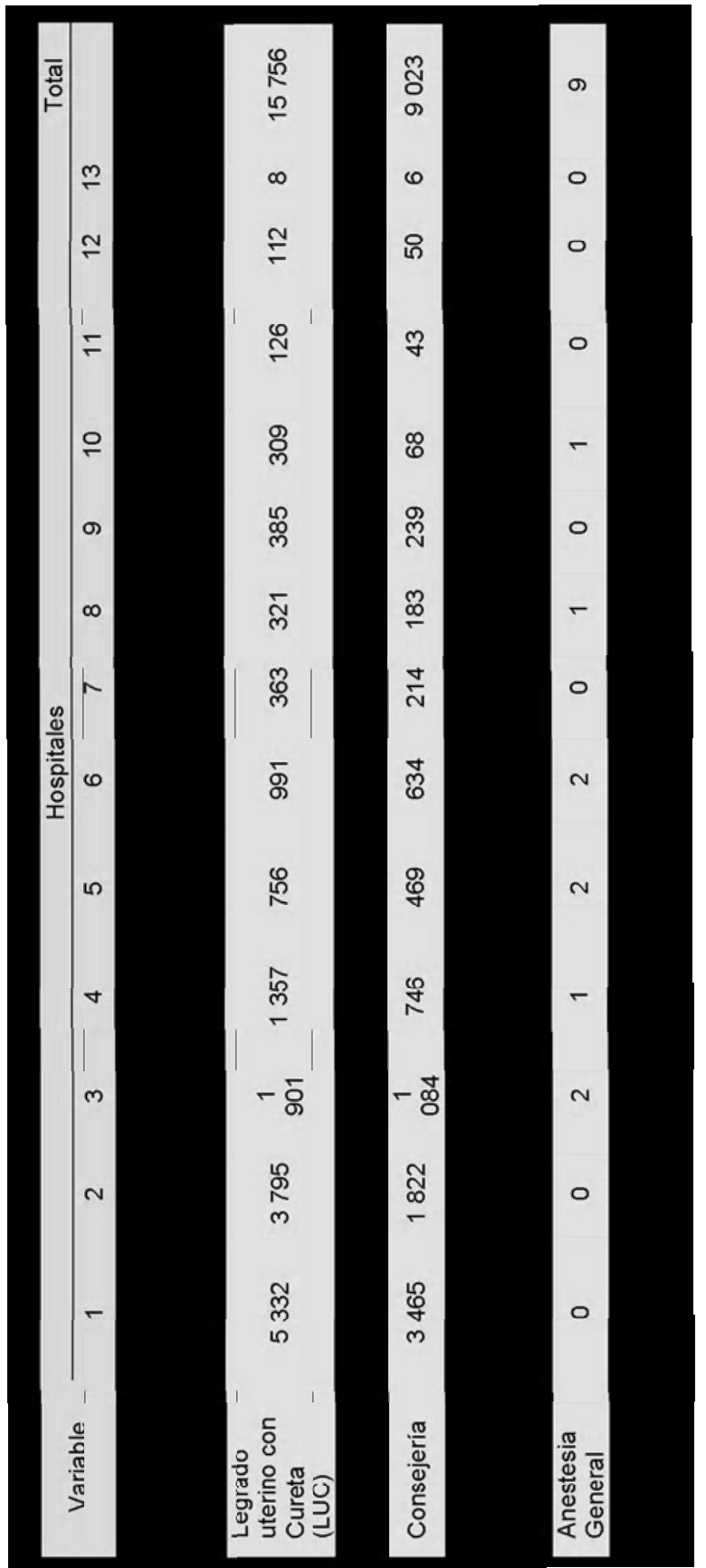


En cuanto a los proveedores se sienten muy comprometidos y ven como propio el programa lo que hace que estén muy pendientes de los registros e informes. Manifestaron en su totalidad satisfacción con el programa APA por las siguientes razones: Una mayor posibilidad de entender los problemas de las mujeres que consultan con abortos incompletos a sus instituciones por tener la oportunidad de un mayor diálogo. En palabras de un proveedor: "antes veíamos un número de historia, un número de cama, ahora tras ese número hay un nombre de una mujer que tiene una vida, una problemática única para ella y me siento en capacidad de brindarle una atención más humana"

Los proveedores conciben que en la atención posaborto de calidad no deben faltar los siguientes elementos: recepción adecuada en el área de emergencia, informar y orientar a las pacientes sobre su diagnóstico y del tratamiento que necesitan; controlar el dolor mediante la administración de los analgésicos y la anestesia requerida para cada caso, con apoyo en la consejería. Facilitar conocimientos sobre salud reproductiva, un sistema de referencia-contrarreferencia eficaz, equipos y materiales adecuados, así como seguimiento a la paciente después que se le dé de alta.

El personal está conforme con su nivel de capacitación, clínica y humana, para el servicio. El 90 \% de los médicos generales aceptan el LUA como una alternativa eficaz en el manejo del aborto incompleto, mientras que dos terceras partes de los ginecobstetras capacitados tienen confianza en el método.

\section{DISCUSION}

El presente estudio mostró un alto grado de satisfacción de las usuarias y de los proveedores con la estrategia de Atención Integral al posaborto (APA), similar a lo observado en países como Honduras (10), Senegal (11) y Filipinas (12).

En cuanto a los componentes del programa se observó que la consejería podría explicar la mejor percepción de la calidad, menor uso de anestésicos generales y mayor satisfacción en las pacientes atendidas con LUA. Lo anterior quizás por mayor apoyo verbal por parte del proveedor(a), facilitando la comunicación con la mujer, explicándole el procedimiento, que va a sentir y porqué y se aprovecha que en la recuperación está despierta para hablar de sus necesidades en SSR, De igual forma la menor estancia hospitalaria con LUA ha podido también ser factor determinante de la mayor satisfacción de las usuarias tratadas con este método. 
En cuanto a la atención del aborto y sus complicaciones actualmente existe consenso en que la técnica de elección para abortos incompletos del primer trimestre es LUA. En la revisión sistemática realizada por la fundación Cochrane (13), al comparar LUA con LUC se encontraron diferencias estadísticamente significativas en: sangrado, dolor y duración del procedimiento. LUA se asoció con menor pérdida sanguínea en el procedimiento (promedio de diferencia -17 mls, 95 \% (IC) -24 a -10 mls), menor dolor (RR): 0.74, 95 \% (IC) 0.61- 0.90) y menor duración del procedimiento (promedio de diferencia -1.2 minutos 95 $\%$ (IC) -1.5 a -0.87 minutos). La literatura internacional ha mostrado que las complicaciones son mayores con LUC $(14,15)$, similar a lo encontrado en este estudio.

A pesar de la evidencia existe aún en nuestro país resistencia a utilizar las nuevas técnicas de evacuación uterina como LUA, especialmente entre los Ginecobstetras; algunos se aferran a la técnica tradicional (LUC) con la que tienen más confianza. Hay que trabajar desde las escuelas formadoras de recurso humano para que al egresado se le den herramientas de actualización y haya menos resistencia a los cambios.

Hay alta dependencia económica de las mujeres de sus compañeros y tienen baja escolaridad lo que debe tenerse en cuenta al momento de la consejería y seguimiento.

La baja frecuencia con que las mujeres fueron informadas sobre doble protección, hace necesario seguir trabajando en la sensibilización de los proveedores para que informen a las mujeres sobre el riesgo de ITS/VIH / SIDA y las formas de protegerse.

La alta frecuencia del uso de algún medicamento para abortar es consistente con lo informado previamente $(16,17)$. Por ende, si bien es cierto el tratamiento médico seguirá siendo la evacuación uterina (medicamentosa, con cureta, con aspiración eléctrica o manual), debemos enfatizar la necesidad de evaluar las necesidades en Salud Sexual y Reproductiva que esa mujer pueda tener, ya que muchas de ellas afrontan una gestación no deseada (independientemente que haya sido un aborto espontáneo o inducido) por ende no debemos permitir que salga de la institución sin haberle explicado sobre su retorno de la fertilidad, opciones anticonceptivas y ojala se retire de la institución con un método anticonceptivo. 
El período posaborto después del tratamiento de emergencia ofrece una importante oportunidad a los profesionales de salud para ayudar a las mujeres resolviendo los problemas que contribuyen al embarazo indeseado (18). Se deberá informar inmediatamente después de la atención a todas las pacientes que han sufrido un aborto sobre el pronto retorno de la ovulación que puede llevar a la posibilidad de un nuevo embarazo en el corto plazo (incluso antes de la primera menstruación posaborto). En abortos espontáneos o inducidos en el primer trimestre la ovulación usualmente sucede entre el día 9 a 11 posaborto. Debe haber disponibilidad en la misma unidad o a través de referencia, de métodos anticonceptivos seguros y eficaces para evitar futuros embarazos indeseados. De igual forma debe haber disponibilidad y recursos locales de servicios de planificación familiar para un reabastecimiento de los métodos, provisión de métodos a largo plazo y permanentes, y atención relacionada continua

Agradecimientos. A los(las) gerentes y trabajadores(as) de las instituciones participantes por su colaboración permanente. A la Fundación Bergstrom que apoyó el programa APA de EngenderHealth en Colombia. A los siguientes profesionales que trabajaron en EngenderHealth y apoyaron decididamente el programa: Rita Cecilia Plata de Silva (directora de la oficina en Colombia), Sally Girvin (primera directora de APA para Latinoamérica) y Lorelei Goodyear (segunda directora de APA para Latinoamérica). AAndrea Eschen (antigua directora regional de EngenderHealth) por sus aporte al programa APA en Colombia. Finalmente agradecemos a Erin Mielke, Coordinadora para Latinoamérica de EngenderHealth por sus valiosos aportes a este documento.

\section{REFERENCIAS}

1. Galvis N, Ochoa A, Rojas A. Frecuencia del aborto incompleto, factores asociados y complicaciones en mujeres adolescentes entre 14 y 19 años que llegan a la sección de gineco-obstetricia de un hospital de tercer nivel en una ciudad de Cuba. Rev. ECM 2003; 8:45-53.

2. Parpinelli MA, Surita F, Pereira BG, Cecatti JG. Mortalidade materna na cidade de Campinas, no período de 1992 a 1994. Rev. Bras. Ginecol. Obstet 1999; 21:227232.

3. Organización Mundial de la salud. Datos y cifras del informe sobre la salud en el mundo 2005. [Internet]. Disponible en: http://www.who.int/whr/2005/media_ centre/facts_figures_es.pdf Consultado: Septiembre 16de 2006).

4. Gómez PI. Salvar la vida de las mujeres: aprovechando oportunidades perdidas. Revista Seguridad Social al día 2003;36:8-10. 
5. Family Care International. Meeting the Cairo Challenge: A Summary Report: Implementing the ICPD Programme of Action. NY: Family Care International: 9; 1999.

6. Ministerio de Protección Social. Colombia. Plan de Choque Mortalidad Materna; Junio 10, 2004.

7. EngenderHealth. Postabortion Care Lessons learned in Latin America. 2003. [Internet]. Disponible en: http://www.engenderhealth.org/pubs/pubslist.html Consultado: Septiembre 14 de 2006.

8. Abernathy M. Planificación de un suministro sostenible del instrumental de aspiración manual endouterina: Una guía para administradores de programas. Carolina del Norte: Chapel Hill. Ipas; 2005.

9. EngenderHealth. Practical guide for selection of MVA instruments. NY, EngenderHealth. 2004. [Internet] Disponible en: http://www.engenderhealth. org/res/offc/pac/mva-guide/pdf/mva_instruments_guide.pdf Consultado: Septiembre 16 de 2006.

10. Population Council. Honduras: Mejorar la salud reproductiva. 2002. [Internet]. Disponible en: http://www.popcouncil.org/pdfs/frontiers/reports/ Honduras_io_mejorar.pdf Consultado: Septiembre 15 de 2006.

11. PRIME II. Senegal: A Community Model for Postabortion Care Services . 2004. [Internet]. Disponible en: http://www.prime2.org/prime2/page/home/47ce21. html?article=372 Consultado: Septiembre 16de 2006).

12. EngenderHealth . Postabortion Care with Compassion in the Philippines. 2005. [Internet]. Disponible en: http://www.engenderhealth.org/itf/philippines.html Consultado: Septiembre 16 de 2006.

13. Forna F, Gülmezoglu AM. Surgical procedures to evacuate incomplete abortion The Cochrane Library, Issue 1. Chichester, UK: John Wiley \& Sons, Ltd.; 2006.

14. Goldberg AB, Dean G, Kang MS, Youssof S, Darney PD. Manual versus electric vacuum aspiration for early first-trimester abortion: a controlled study of complication rates. Obstet Gynecol. 2004; 103:101-7.

15. Billings D, Fuentes J, Pérez-Cuevas R. Comparing the Quality of Three Models of Postabortion Care in Public Hospitals in Mexico City. International Family Planning Perspectives, 2003; 29: 112-120

16. Sherris BA, Burns MA, Girvin S, Westley E, Gómez PI. Misoprostol use in developing countries: results from a multicountry study. International Journal of Gynecology and Obstetrics 2005; 88: 76-81.

17. Henshaw S, Singh S, Haas T. La Incidencia del Aborto Inducido a Nivel Mundial. Perspectivas Internacionales en Planificación Familiar, número especial.1999; 16-24.

18. Gómez PI. Atención Integral al postaborto, Experiencia Latinoamericana. En Libro Obstetricia y Ginecología en el próximo milenio, FLASOG, El Salvador. 1999:339. 